\title{
Laplace DLTS study of deep defects created in neutron-irradiated n-type $4 \mathrm{H}-$ $\mathrm{SiC}$
}

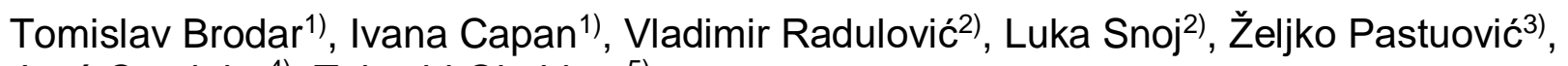
José Coutinho ${ }^{4)}$, Takeshi Ohshima ${ }^{5}$

1) Division of Materials Physics, Ruđer Bošković Institute, Bijenicka 54, 10000 Zagreb, Croatia,

2) Reactor Physics Division, Jožef Stefan Institute, Jamova 39, 1000 Ljubljana, Slovenia,

3) Centre for Accelerator Science, Australian Nuclear Science and Technology Organisation, 1 New Illawarra Rd, Lucas Heights NSW 2234, Australia,

4) Department of Physics and I3N, University of Aveiro, Campus Santiago, 3810-193 Aveiro, Portugal,

5) Takasaki Advanced Radiation Research Institute, National Institutes for Quantum and Radiological Science and Technology, 1233 Watanuki, Takasaki, Gunma 3701292, Japan

This paper presents the characterization of the electrically active defects created by epithermal and fast neutrons in epitaxial n-type $4 \mathrm{H}-\mathrm{SiC}$ material using Laplace Deep Level Transient Spectroscopy (Laplace DLTS). While the deep level related to the carbon vacancy has been observed in as-grown material, we observed that epithermal and fast neutron irradiation introduces additional simple defect complexes, with energy levels at $\mathrm{E}_{\mathrm{C}}-0.40 \mathrm{eV}$ and $\mathrm{E}_{\mathrm{C}}-0.70 \mathrm{eV}$.

Keywords: Epitaxial n-type 4H-SiC, Laplace DLTS, defects, neutron irradiation.

Corresponding author: Tomislav Brodar, tbrodar@irb.hr 


\section{Introduction}

Silicon carbide $(\mathrm{SiC})$ is a radiation-hard wide band gap semiconductor suitable for high temperature, high-frequency and high-power applications [1,2]. Due to the high and isotropic mobility of carriers, the $4 \mathrm{H}$ polytype of $\mathrm{SiC}$ is preferred as material for radiation detector applications [3]. Neutron radiation detectors based on SiC have attracted considerable interest in recent years [4].

Characterization of defects created by ionizing radiation in n-type epitaxial $4 \mathrm{H}-\mathrm{SiC}$ layers is crucial for future improvement of radiation hardness and extending the lifetime of $4 \mathrm{H}$ $\mathrm{SiC}$ detectors by material engineering. Electrically active defects influence the electrical properties of the semiconductor and in general, cause a deterioration of the detector spectroscopic performance. This is essentially due to recombination of charge carriers at deep levels, which decrease the minority carrier lifetime, and consequently the charge collection efficiency of the detector. An increase in the concentration of a suitable impurity can suppress the formation of the most prominent/influential deep levels and increase the detection efficiency $[5,6]$.

Numerous DLTS studies were conducted on $4 \mathrm{H}-\mathrm{SiC}$ after controlled introduction of defects in the material by electron and proton irradiation or ion implantation. The dominating deep levels in as-grown n-type $4 \mathrm{H}-\mathrm{SiC}$ are $\mathrm{Z}_{1 / 2}$ and $\mathrm{EH}_{6 / 7}$. The $\mathrm{Z}_{1 / 2}$ deep level has been assigned to double acceptor state transition of carbon vacancy $\mathrm{V}_{\mathrm{C}}(=/ 0)$ [7], while the $\mathrm{EH}_{6 / 7}$ deep level has been assigned to donor state of carbon vacancy. It has been shown that the $Z_{1 / 2}$ peak consists of two peaks $Z_{1}(=/ 0)$ and $Z_{2}(=/ 0)$ assigned to carbon vacancies on two different lattice sites $V_{C}(h)$ and $V_{C}(k)$ [8]. Negative- $U$ ordering of $Z_{1}(=/ 0)$ and $Z_{2}(=/ 0)$ has been observed by DLTS measurements and density functional theory (DFT) calculations $[9,10]$.

The assignment of other commonly observed deep levels in irradiated n-type $4 \mathrm{H}-\mathrm{SiC}$ is still uncertain and only speculations are possible with the available data. Two deep levels usually labeled as S1/S2 [11,12], S2/S4 [13,14] or EH1/EH3 [15-18] with energies around $\mathrm{E}_{\mathrm{C}}-0.4 \mathrm{eV}$ and $\mathrm{E}_{\mathrm{C}}-0.7 \mathrm{eV}[19,20]$ can be observed in $\mathrm{n}$-type $4 \mathrm{H}-\mathrm{SiC}$ after electron or proton irradiation and ion implantation. These defects are introduced by low energy electron irradiation which displaces only the carbon atom in the $4 \mathrm{H}-\mathrm{SiC}$ crystal lattice [15]. Low temperature annealing studies have indicated that these are defects related to carbon interstitials $[13,21]$ and that the decrease in their concentration is correlated with an increase in the charge collection efficiency [17]. These defects have been investigated mostly in electron or proton irradiated samples, however neutron irradiation induced defects have not been studied exhaustively $[22,23]$.

Although the $\mathrm{S}$ and $\mathrm{EH} 1 / \mathrm{EH} 3$ radiation induced defects have usually been attributed to $\mathrm{C}$ displacements (displacement threshold $20 \mathrm{eV}[15,24]$ ), particularly after low energy electron irradiation, with more energetic fast neutron irradiation additional defects are 
expected. The probability for silicon displacement (displacement threshold $35 \mathrm{eV}[15,24]$ ) increases with the energy of incident particle.

In this paper, we report on Laplace DLTS measurements of neutron irradiated $4 \mathrm{H}-\mathrm{SiC}$ Schottky barrier diodes. Laplace DLTS, which offers an order of magnitude better energy resolution compared to conventional DLTS, has been successfully applied in resolving the broad DLTS peak, known as $Z_{1 / 2}$ into two components, $Z_{1}(=/ 0)$ and $Z_{2}(=/ 0)$. Moreover, Laplace DLTS was applied for the estimation of activation energies for electron emission from defects introduced by epithermal and fast neutron irradiation. Interest in these defects is due to their influence on the efficiency and lifetime of radiation hard neutron detectors.

\section{Materials and Methods}

n-type SiC Schottky barrier diodes (SBDs) were produced on nitrogen-doped (up to $4.5 \times 10^{14} \mathrm{~cm}^{-3}$ ), epitaxially grown $4 \mathrm{H}-\mathrm{SiC}$ single crystal layers, approximately $25 \mu \mathrm{m}$ thick [25]. The Schottky barrier was formed by evaporation of nickel through a metal mask with patterned square apertures of $1 \mathrm{~mm} \times 1 \mathrm{~mm}$, while Ohmic contacts were formed on the backside of the $\mathrm{SiC}$ substrate by nickel sintering at $950^{\circ} \mathrm{C}$ in $\mathrm{Ar}$ atmosphere.

The produced SBDs were irradiated with epithermal and fast neutrons at the Jožef Stefan Institute (JSI) TRIGA reactor in Ljubljana, Slovenia. Thermal neutrons with energy below $0.55 \mathrm{eV}$ were filtered by irradiating the Schottky barrier diodes inside a cadmium box with a wall thickness of $1 \mathrm{~mm}$. The neutron energy spectrum in the irradiation location was characterized on the basis of Monte Carlo calculations with the MCNP® code [26] and activation measurements [27]. The total neutron flux in the irradiation location was monitored by activation measurements for the ${ }^{197} \mathrm{Au}(\mathrm{n}, \mathrm{\gamma})$ reaction for each used power level. The sub-cadmium neutron flux was derived from the characterized neutron spectrum, the cut-off energy of the cadmium box $(0.55 \mathrm{eV})$ and the total neutron flux. The fractions of the epithermal neutron flux (neutron energies in the interval between $0.55 \mathrm{eV}$ and $100 \mathrm{keV}$ ) and the fast neutron flux (neutron energies in the interval between $100 \mathrm{keV}$ and $20 \mathrm{MeV}$ ) are approximately $51 \%$ and $49 \%$. The epithermal range displays typical $1 / E$ behavior with negligible slope $\left(\alpha \approx 0\right.$ in the parametrization $\left.\varphi(E) \sim 1 / E^{1+\alpha}\right)$ and some small peaks and dips due to resonance absorption effects. The fast component follows a typical fission spectrum, with a peak region from around $1 \mathrm{MeV}$ to around $2 \mathrm{MeV}$.

Only intrinsic defects are introduced in $4 \mathrm{H}-\mathrm{SiC}$ by displacement of silicon and carbon atoms. Samples were irradiated at room temperature. The specified neutron fluence levels for the sample irradiations spanned several orders of magnitude, in the range from $10^{9} \mathrm{n} / \mathrm{cm}^{2}$ up to $10^{14} \mathrm{n} / \mathrm{cm}$. Table 1 reports the reactor power level and the irradiation time for each irradiated sample.

Temperature dependent current-voltage (I-V) and $1 \mathrm{MHz}$ capacitance-voltage (C-V) measurements were carried out using a Keithley 6487 Picoammeter/Voltage Source 
and a Keithley 4200 Semiconductor characterization system. Laplace DLTS and DLTS measurements were carried out using an experimental set up consisting of a Boonton 7200 capacitance meter, NI PCI-6251 DAQ and Laplace DLTS software [28]. Laplace DLTS and DLTS measurements were performed in the temperature range from 130 up to $380 \mathrm{~K}$. Laplace DLTS spectra with 200 points were calculated by the FLOG numerical routine [29] for all samples. Capacitance transients used as the input of FLOG numerical routine were measured with sampling rates in the range from $10 \mathrm{kHz}$ up to $70 \mathrm{kHz}$, number of points $\sim 3 \times 10^{4}$ and number of averaged transients in the range from 200 up to 1000. Prior to the measurements, the samples were cooled down from room temperature with no bias voltage applied.

Capacitance amplitudes of deep levels from Laplace DLTS and DLTS measurements and values of capacitance from $\mathrm{C}-\mathrm{V}$ measurements at reverse bias and pulse bias were used for calculation of concentrations of deep levels [30]. The Lambda effect was not taken into account.

\section{Results and Discussion}

The prepared $4 \mathrm{H}-\mathrm{SiC}$ SBDs showed excellent rectifying properties before neutron irradiation. The measured I-V characteristics showed a reverse current at $300 \mathrm{~K}$ of less than $10 \mathrm{pA}$ at $-10 \mathrm{~V}$ for all samples. An increase in the series resistance of the Schottky diodes was observed from forward I-V characteristics at neutron fluences higher than $10^{13} \mathrm{n} / \mathrm{cm}^{2}$, in agreement with other results from the literature [22,31]. The series resistance influenced only DLTS measurements of samples FN5E13 and FN1E14, while the other samples had negligible series resistance.

We characterized deep levels introduced by epithermal and fast neutron irradiation using DLTS measurements performed in the temperature range up to $380 \mathrm{~K}$ (Fig. 1). We observed only one broad peak with maximum at around $300 \mathrm{~K}$ in the non-irradiated sample $F N 0$. This peak is already reported, it is known as $Z_{1 / 2}$ and it is assigned to the carbon vacancy $\mathrm{V}_{\mathrm{C}}(=/ 0)$ [7]. Epithermal and fast neutron irradiation with fluence up to $10^{11} \mathrm{n} / \mathrm{cm}^{2}$ did not cause any new observable defects in the DLTS spectra. Peaks labeled $\mathrm{EH} 1$ and EH3 appear for higher neutron fluences and can be observed in the DLTS spectra of samples FN1E12 and FN1E13. Deep levels EH1 and EH3 [15] (or S1 and S2 $[12]$ or S2 and S4 $[13,14]$ ) already observed after the low energy electron irradiation and ion implantation, respectively match the $\mathrm{EH} 1$ and $\mathrm{EH} 3$ deep levels on Fig. 1

The $Z_{1 / 2}$ peak observed in DLTS spectra was clearly resolved into two peaks $Z_{2}(=/ 0)$ and $Z_{1}(=/ 0)$ by Laplace DLTS measurements on as-grown FN0 sample (Fig. 2). $Z_{1}(=/ 0)$ and $\mathrm{Z}_{2}(=/ 0)$ have been assigned to the carbon vacancy on the hexagonal $\mathrm{V}_{\mathrm{C}}(\mathrm{h})$ and the cubic $\mathrm{V}_{\mathrm{C}}(\mathrm{k})$ site, respectively. Laplace DLTS spectra at $300 \mathrm{~K}$ of FN1E12 and FN1E13 samples might contain introduced deep levels with emissions close to $Z_{1}(=/ 0)$ or $Z_{2}(=/ 0)$. Therefore, Laplace DLTS analyses of $Z_{1}(=/ 0)$ and $Z_{2}(=/ 0)$ at higher fluences than $10^{12} \mathrm{n} / \mathrm{cm}^{2}$ became ambiguous and the uncertainty of their calculated emissions increased, as the width of the $Z_{1}(=/ 0)$ and $Z_{2}(=/ 0)$ peaks is too broad for Laplace DLTS measurement analysis. At this stage, we can only speculate that the broadening of the $Z_{1}(=/ 0)$ and $Z_{2}(=/ 0)$ peaks in 
Laplace DLTS spectra of samples irradiated with high neutron fluences could be caused either by the broader emission distribution of the introduced carbon vacancies perturbed by a nearby defects or the introduction of additional unresolved deep levels.

We investigated the influence of neutron irradiation on the free carrier concentration and acceptor levels $\left(Z_{1}(=/ 0)\right.$ and $\left.Z_{2}(=/ 0)\right)$ concentrations as a function of neutron fluence (Fig. $3)$. Using Laplace DLTS, we estimated the concentration of $Z_{1}(=/ 0)$ and $Z_{2}(=/ 0)$ as individual deep levels for the first time. Epithermal and fast neutron irradiation did not cause changes in the free carrier concentration up to the neutron fluence of $10^{12} \mathrm{n} / \mathrm{cm}^{2}$, which confirms the expected, i.e. the excellent radiation hardness of $4 \mathrm{H}-\mathrm{SiC}$ material. The significant decrease in the free carrier concentration is accompanied with the increase in the concentration of the $Z_{1}(=/ 0)$ and $Z_{2}(=/ 0)$ concentrations for fluence levels higher than $10^{12} \mathrm{n} / \mathrm{cm}^{2}$. The decrease in the free carrier concertation $n$ as a function of the neutron fluence $\Phi$ is well described by a linear equation [33]:

$$
\mathrm{n}(\Phi)=\mathrm{n}_{0}-\mathrm{K} \Phi,
$$

where the $n_{0}$ is the initial doping concentration and $K$ is the removal rate. The calculated removal rate for epithermal and fast neutron irradiation is $6.8 \mathrm{~cm}^{-1}$ and it is of the same order of magnitude as previously reported values for neutron irradiation. Differences in the removal rates are possibly due to different irradiation parameters or neutron energy spectra [34].

Laplace DLTS measurements (Fig. 4 and Fig. 5) on epithermal and fast neutron irradiated samples did not reveal the formation of additional deep levels overlapping with $\mathrm{EH} 1$ and $\mathrm{EH}$. As already mentioned, irradiations and ion implantations performed at sufficiently high energies for displacement of silicon atoms could potentially introduce additional deep levels in DLTS spectra overlapping with deep levels related to carbon displacement. In the case of alpha particle irradiation a peak which overlaps with $\mathrm{EH} 1$ has been observed in DLTS spectrum [20].

The values for activation energies for electron emission, apparent electron capture cross sections and introduction rates are determined for all defects by Laplace DLTS (Table 2). Introduction rates for the $Z_{1}(=/ 0)$ and $Z_{2}(=/ 0)$ as individual defects have been directly measured for the first time using Laplace DLTS.

The introduction rate for $\left.Z_{2}=/ 0\right)$ is approximately twice the $Z_{1}(=/ 0)$ introduction rate, while their concentration ratio $Z_{2}(=/ 0): Z_{1}(=/ 0)$ is $\approx 4: 1$ in the as-grown FN0 sample. This result agrees well with DFT calculations where it was found that carbon vacancy in the double negative charge state is more stable on a lattice site with local cubic symmetry $V_{C}(k,=)$ assigned to $Z_{2}(=/ 0)$, compared to the hexagonal lattice site $V_{C}(h,=)$ assigned to $Z_{1}(=/ 0)$ [36]. It is not clear though, why upon irradiation, the introduction rate of cubic $V_{C}$ defects is greater than that of hexagonal $V_{C}$ defects.

Introduction rates of observed defects after epithermal fast neutron irradiation are of the same order of magnitude as the $\mathrm{EH} 1, \mathrm{Z}_{1 / 2}$ and $\mathrm{EH} 3$ introduction rates in the case of electron irradiation $[13,14]$ and an order of magnitude lower than in the case of proton irradiation $[14,37]$. The highest introduction rate after neutron irradiation is observed for 
the $\mathrm{EH} 3$ deep level, contrary to the electron and proton irradiation where $Z_{1 / 2}$ has the highest introduction rate.

Our results for the introduction rates of $\mathrm{EH} 1$ and $\mathrm{EH} 3$ support the assignment of the observed deep levels to simple defect complexes as epithermal and fast neutron irradiation introduces them with concentrations ratios comparable with electron and proton irradiations. There are several defects which could be observed by Laplace DLTS spectra in the temperature range up to $380 \mathrm{~K}$ if annealing studies [15] and DFT calculations are considered [10,38-43]. The simplest deep defects among them are $\mathrm{C}_{\mathrm{i}}(-$ 10) [42], $\left(\mathrm{C}_{\mathrm{i}}\right)_{2}(-/ 0)$ [38] and $\mathrm{Si}_{\mathrm{i}}(-/ 0)$ [43]. Considering the results on neutron irradiation and the deep defects presented in this study, we tentatively assign $\mathrm{EH} 1$ and $\mathrm{EH} 3$ to carbon displacements, most probably to $\mathrm{C}_{\mathrm{i}}$-related defects or complexes.

\section{Conclusions}

This paper reports results of Laplace DLTS measurements performed on epithermal and fast neutron irradiated $n$-type $4 \mathrm{H}$-SiC Schottky barrier diodes. The $Z_{1 / 2}$ peak observed in the conventional DLTS spectra was clearly resolved into two peaks $Z_{2}(=/ 0)$ and $Z_{1}(=/ 0)$ by Laplace DLTS. Neutron irradiation introduced two additional deep levels, EH1 and $\mathrm{EH} 3$, which were assigned to simple defect complexes. Their activation energies, apparent capture cross sections and introduction rates were determined by Laplace DLTS measurements.

\section{Acknowledgments}

This work is supported by the NATO SPS programme [project number 985215]. JC would like to thank the support by the Fundação para a Ciência e a Tecnologia (FCT) [project UID/CTM/50025/2013]. IC would like to thank financial support from the European Regional Development Fund for the 'Center of Excellence for Advanced Materials and Sensing Devices' [Grant No. KK.01.1.1.01.0001]. ZP would like to acknowledge the NCRIS funding provided by the Australian Government. The JSI project team would like to acknowledge the support from the Slovenian Ministry of Education, Science and Sport [project no. P2-0073 - Reactor Physics].

\section{References}

[1] R. Hedayati, L. Lanni, S. Rodriguez, B.G. Malm, A. Rusu, C.M. Zetterling, A monolithic, $500{ }^{\circ} \mathrm{C}$ operational amplifier in $4 \mathrm{H}-\mathrm{SiC}$ bipolar technology, IEEE Electron Device Lett. 35 (2014) 693-695. doi:10.1109/LED.2014.2322335.

[2] P. Neudeck, R. Okojie, L. Chen, High-temperature electronics-a role for wide bandgap semiconductors?, Proc. IEEE. 90 (2002) 1065-1076. doi:10.1109/JPROC.2002.1021571.

[3] P.J. Sellin, J. Vaitkus, New materials for radiation hard semiconductor dectectors, Nucl. Instruments Methods Phys. Res. Sect. A Accel. Spectrometers, Detect. Assoc. Equip. 557 (2006) 479-489. doi:10.1016/j.nima.2005.10.128. 
[4] V. Radulović, A. Klemen, L. Snoj, I. Capan, T. Brodar, Z. Ereš, Ž. Pastuović, A. Sarbutt, T. Ohshima, Y. Yamazaki, J. Coutinho, E-SiCure Collaboration Project: Silicon Carbide Material Studies and Detector Prototype Testing at the JSI TRIGA Reactor, in: 27th International Conference Nuclear Energy for New Europe, Portorož, 2018.

[5] H.M. Ayedh, R. Nipoti, A. Hallén, B.G. Svensson, Elimination of carbon vacancies in $4 \mathrm{H}$-SiC employing thermodynamic equilibrium conditions at moderate temperatures, Appl. Phys. Lett. 107 (2015) 252102. doi:10.1063/1.4938242.

[6] L. Storasta, H. Tsuchida, T. Miyazawa, T. Ohshima, Enhanced annealing of the Z12 defect in 4H-SiC epilayers, J. Appl. Phys. 103 (2008) 013705. doi:10.1063/1.2829776.

[7] N.T. Son, X.T. Trinh, L.S. Løvlie, B.G. Svensson, K. Kawahara, J. Suda, T. Kimoto, T. Umeda, J. Isoya, T. Makino, T. Ohshima, E. Janzén, Negative-U system of carbon vacancy in 4H-SiC, Phys. Rev. Lett. 109 (2012). doi:10.1103/PhysRevLett.109.187603.

[8] I. Capan, T. Brodar, Z. Pastuović, R. Siegele, T. Ohshima, S.I. Sato, T. Makino, L. Snoj, V. Radulović, J. Coutinho, V.J.B. Torres, K. Demmouche, Double negatively charged carbon vacancy at the $\mathrm{h}$ - and k-sites in 4H-SiC: Combined Laplace-DLTS and DFT study, J. Appl. Phys. 123 (2018). doi:10.1063/1.5011124.

[9] C.G. Hemmingsson, N.T. Son, A. Ellison, J. Zhang, E. Janzén, Negative- U centers in 4 H silicon carbide, Phys. Rev. B. 58 (1998) R10119-R10122. doi:10.1103/PhysRevB.58.R10119.

[10] T. Hornos, A. Gali, B.G. Svensson, Large-Scale Electronic Structure Calculations of Vacancies in $4 \mathrm{H}-\mathrm{SiC}$ Using the Heyd-Scuseria-Ernzerhof Screened Hybrid Density Functional, Mater. Sci. Forum. 679-680 (2011) 261-264. doi:10.4028/www.scientific.net/MSF.679-680.261.

[11] M.L. David, G. Alfieri, E.M. Monakhov, A. Hallén, C. Blanchard, B.G. Svensson, J.F. Barbot, Electrically active defects in irradiated $4 \mathrm{H}-\mathrm{SiC}$, J. Appl. Phys. 95 (2004) 4728-4733. doi:10.1063/1.1689731.

[12] G. Alfieri, E. V. Monakhov, B.G. Svensson, M.K. Linnarsson, Annealing behavior between room temperature and $2000{ }^{\circ} \mathrm{C}$ of deep level defects in electronirradiated n-type 4H silicon carbide, J. Appl. Phys. 98 (2005) 043518. doi:10.1063/1.2009816.

[13] A. Castaldini, A. Cavallini, L. Rigutti, F. Nava, Low temperature annealing of electron irradiation induced defects in 4H-SiC, Appl. Phys. Lett. 85 (2004) 37803782. doi:10.1063/1.1810627.

[14] A. Castaldini, A. Cavallini, L. Rigutti, F. Nava, S. Ferrero, F. Giorgis, Deep levels by proton and electron irradiation in 4H-SiC, J. Appl. Phys. 98 (2005) 053706. doi:10.1063/1.2014941.

[15] L. Storasta, J.P. Bergman, E. Janzén, A. Henry, J. Lu, Deep levels created by low 
energy electron irradiation in 4H-SiC, J. Appl. Phys. 96 (2004) 4909-4915. doi:10.1063/1.1778819.

[16] C. Hemmingsson, N.T. Son, O. Kordina, J.P. Bergman, E. Janzén, J.L. Lindström, S. Savage, N. Nordell, Deep level defects in electron-irradiated $4 \mathrm{H} \mathrm{SiC}$ epitaxial layers, J. Appl. Phys. 81 (1997) 6155-6159. doi:10.1063/1.364397.

[17] N. Iwamoto, B.C. Johnson, N. Hoshino, M. Ito, H. Tsuchida, K. Kojima, T. Ohshima, Defect-induced performance degradation of $4 \mathrm{H}-\mathrm{SiC}$ Schottky barrier diode particle detectors, J. Appl. Phys. 113 (2013) 143714. doi:10.1063/1.4801797.

[18] K. Kawahara, X. Thang Trinh, N. Tien Son, E. Janzén, J. Suda, T. Kimoto, Investigation on origin of $\mathrm{Z} 1 / 2$ center in $\mathrm{SiC}$ by deep level transient spectroscopy and electron paramagnetic resonance, Appl. Phys. Lett. 102 (2013) 112106. doi:10.1063/1.4796141.

[19] A.T. Paradzah, F.D. Auret, M.J. Legodi, E. Omotoso, M. Diale, Electrical characterization of $5.4 \mathrm{MeV}$ alpha-particle irradiated $4 \mathrm{H}-\mathrm{SiC}$ with low doping density, Nucl. Instruments Methods Phys. Res. Sect. B Beam Interact. with Mater. Atoms. 358 (2015) 112-116. doi:10.1016/j.nimb.2015.06.006.

[20] E. Omotoso, W.E. Meyer, F.D. Auret, A.T. Paradzah, M.J. Legodi, Electrical characterization of deep levels created by bombarding nitrogen-doped $4 \mathrm{H}-\mathrm{SiC}$ with alpha-particle irradiation, Nucl. Instruments Methods Phys. Res. Sect. B Beam Interact. with Mater. Atoms. 371 (2016) 312-316. doi:10.1016/j.nimb.2015.09.084.

[21] F. Gao, W.J. Weber, M. Posselt, V. Belko, Atomic Computer Simulations of Defect Migration in 3C and 4H-SiC, Mater. Sci. Forum. 457-460 (2004) 457-460. doi:10.4028/www.scientific.net/MSF.457-460.457.

[22] F. Nava, A. Castaldini, A. Cavallini, P. Errani, V. Cindro, Radiation detection properties of $4 \mathrm{H}-\mathrm{SiC}$ schottky diodes irradiated up to $1016 \mathrm{n} / \mathrm{cm} 2$ by $1 \mathrm{MeV}$ neutrons, IEEE Trans. Nucl. Sci. 53 (2006) 2977-2982. doi:10.1109/TNS.2006.882777.

[23] E. V Kalinina, G.F. Kholuyanov, D. V Davydov, A.M. Strel'chuk, A. Hallen, A.O. Konstantinov, V. V Luchinin, A.Y. Nikiforov, Effect of irradiation with fast neutrons on electrical characteristics of devices based on CVD 4H-SiC epitaxial layers, Semiconductors. 37 (2003) 1229-1233. doi:10.1134/1.1619523.

[24] J.W. Steeds, F. Carosella, a. G. Evans, M.M. Ismail, L.R. Danks, W. Voegeli, Differentiation between $\mathrm{C}$ and Si Related Damage Centres in $4 \mathrm{H}$ - and $6 \mathrm{H}-\mathrm{SiC}$ by the Use of 90-300 kV Electron Irradiation Followed by Low Temperature Photoluminescence Microscopy, Mater. Sci. Forum. 353-356 (2001) 381-384. doi:10.4028/www.scientific.net/MSF.353-356.381.

[25] M. Ito, L. Storasta, H. Tsuchida, Development of 4H-SiC Epitaxial Growth Technique Achieving High Growth Rate and Large-Area Uniformity, Appl. Phys. Express. 1 (2008) 015001. doi:10.1143/APEX.1.015001. 
[26] T. Goorley, M. James, T. Booth, F. Brown, J. Bull, L.J. Cox, J. Durkee, J. Elson, M. Fensin, R.A. Forster, J. Hendricks, H.G. Hughes, R. Johns, B. Kiedrowski, R. Martz, S. Mashnik, G. McKinney, D. Pelowitz, R. Prael, J. Sweezy, L. Waters, T. Wilcox, T. Zukaitis, Initial MCNP6 Release Overview, Nucl. Technol. 180 (2012) 298-315. doi:10.13182/NT11-135.

[27] A. Trkov, V. Radulović, L. Snoj, The GRUPINT neutron spectrum adjustment code - general features and characterization of the spectra in three irradiation channels of the JSI TRIGA reactor, in: 16th Int. Symp. React. Dosim., Program and Book of abstracts, Santa Fe, USA, 2017.

[28] Laplace DLTS software, (2018). http://info.ifpan.edu.pl/Dodatki/WordPress/laplacedlts/.

[29] L. Dobaczewski, A.R. Peaker, K. Bonde Nielsen, Laplace-transform deep-level spectroscopy: The technique and its applications to the study of point defects in semiconductors, J. Appl. Phys. 96 (2004) 4689-4728. doi:10.1063/1.1794897.

[30] Blood P., Orton J. W., The Electrical Characterization of Semiconductors Majority Carriers and Electron States (Techniques of Physics), Academic Press, 1992.

[31] L. Zhang, Y. Zhang, Y. Zhang, C. Han, Neutron radiation effect on 4H-SiC MESFETs and SBDs, J. Semicond. 31 (2010) 114006. doi:10.1088/16744926/31/11/114006.

[32] T. Brodar, I. Capan, V. Radulović, L. Snoj, Ž. Pastuović, J. Coutinho, T. Ohshima, C-V measurements on the n-type $4 \mathrm{H}-\mathrm{SiC}$ Schottky barrier diodes, (2018). doi:10.17632/m9mbdxsf6x.1.

[33] B. Novoselnik, M. Pilipovic, R. Jacimovic, B. Pivac, R. Slunjski, I. Capan, Capacitance changes in neutron irradiated n-type silicon: The flux effect, Nucl. Instruments Methods Phys. Res. Sect. B Beam Interact. with Mater. Atoms. 268 (2010) 2400-2402. doi:10.1016/j.nimb.2010.04.017.

[34] A.A. Lebedev, Deep level centers in silicon carbide: A review, Semiconductors. 33 (1999) 107-130. doi:10.1134/1.1187657.

[35] C. Claeys, E. Simoen, Radiation Effects in Advanced Semiconductor Materials and Devices, Springer, Berlin, 2002. doi:10.1007/978-3-662-04974-7.

[36] J. Coutinho, V.J.B. Torres, K. Demmouche, S. Öberg, Theory of the carbon vacancy in 4H -SiC: Crystal field and pseudo-Jahn-Teller effects, Phys. Rev. B. 96 (2017) 1-16. doi:10.1103/PhysRevB.96.174105.

[37] A. Castaldini, A. Cavallini, L. Rigutti, Assessment of the intrinsic nature of deep level Z 1 / Z 2 by compensation effects in proton-irradiated $4 \mathrm{H}-\mathrm{SiC}$, Semicond. Sci. Technol. 21 (2006) 724-728. doi:10.1088/0268-1242/21/6/002.

[38] A. Gali, N.T. Son, E. Janzén, Electrical characterization of metastable carbon clusters in SiC: A theoretical study, Phys. Rev. B - Condens. Matter Mater. Phys. 73 (2006). doi:10.1103/PhysRevB.73.033204. 
[39] F. Devynck, A. Alkauskas, P. Broqvist, A. Pasquarello, Charge transition levels of carbon-, oxygen-, and hydrogen-related defects at the $\mathrm{SiC} / \mathrm{SiO} 2$ interface through hybrid functionals, Phys. Rev. B - Condens. Matter Mater. Phys. 84 (2011). doi:10.1103/PhysRevB.84.235320.

[40] T. Umeda, N.T. Son, J. Isoya, E. Janzén, T. Ohshima, N. Morishita, H. Itoh, A. Gali, M. Bockstedte, Identification of the carbon antisite-vacancy pair in $4 \mathrm{H}-\mathrm{SiC}$, Phys. Rev. Lett. 96 (2006) 14-17. doi:10.1103/PhysRevLett.96.145501.

[41] M. Bockstedte, A. Mattausch, O. Pankratov, Ab initio study of the annealing of vacancies and interstitials in cubic SiC: Vacancy-interstitial recombination and aggregation of carbon interstitials, Phys. Rev. B - Condens. Matter Mater. Phys. 69 (2004) 1-13. doi:10.1103/PhysRevB.69.235202.

[42] F. Devynck, A. Alkauskas, P. Broqvist, A. Pasquarello, M. Caldas, N. Studart, Energy levels of candidate defects at SiCSiO2 interfaces, Energy. 108 (2010) 108-109. doi:10.1063/1.3295319.

[43] T. Hornos, N.T. Son, E. Janzén, A. Gali, Theoretical study of small silicon clusters in 4H-SiC, Phys. Rev. B. 76 (2007) 165209. doi:10.1103/PhysRevB.76.165209. 


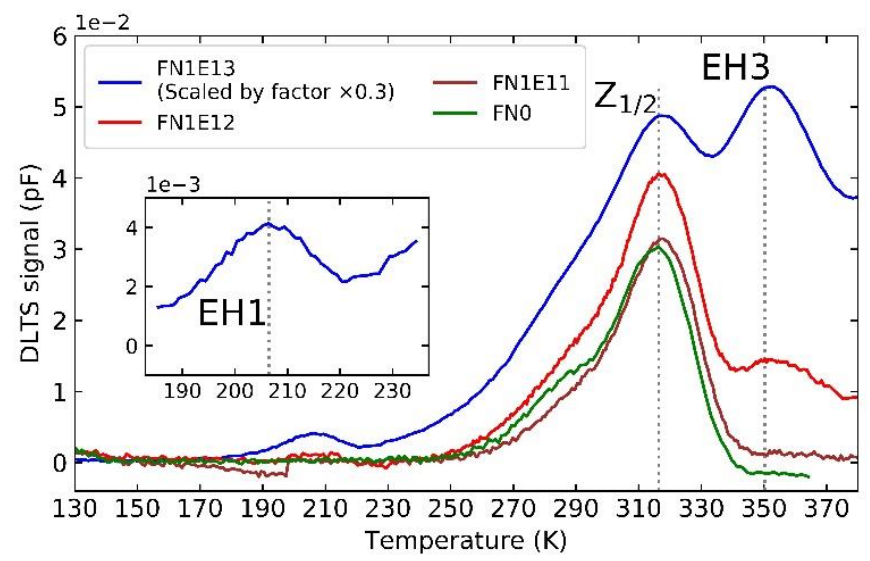

Fig. 1. DLTS spectra of the as grown sample and neutron irradiated samples (emission rate $50 \mathrm{~s}^{-1}$ ) in temperature range from $130 \mathrm{~K}$ up to $380 \mathrm{~K}$. Voltage settings are reverse bias $V_{R}=-10 \mathrm{~V}$, pulse bias $V_{P}=-0.1 \mathrm{~V}$ and pulse width $t_{p}=10 \mathrm{~ms}$. Magnified DLTS spectra of FN1E13 sample is shown in the inset.

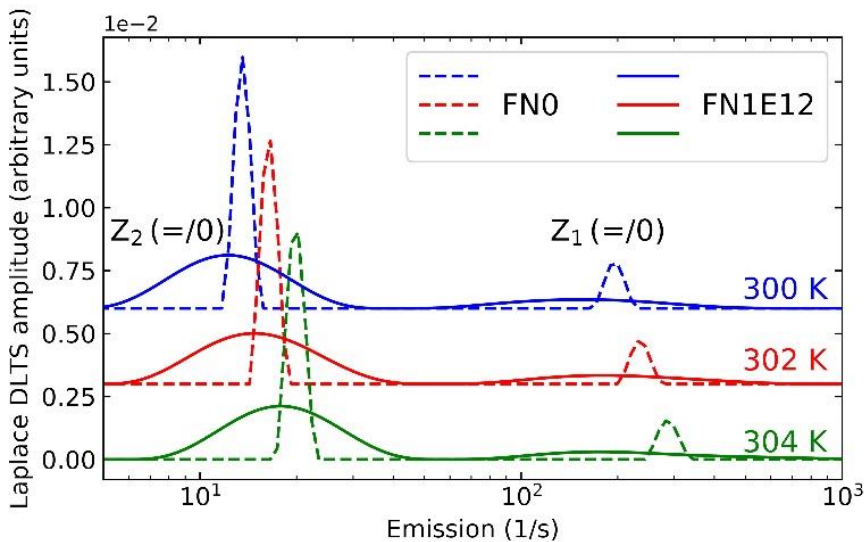

Fig. 2. Laplace DLTS spectra of the as-grown FNO and FN1E12 samples at three subsequent measurement temperatures. Laplace DLTS measurements were performed on the sample FNO in temperature range from $282 \mathrm{~K}$ up to $304 \mathrm{~K}$ with $2 \mathrm{~K}$ step and on the sample FN1E12 in temperature range from $270 \mathrm{~K}$ up to $318 \mathrm{~K}$ with $2 \mathrm{~K}$ step. Voltage settings are reverse bias $V_{R}=-10 \mathrm{~V}$, pulse bias $V_{P}=-0.1 \mathrm{~V}$, pulse width $t_{p}=10 \mathrm{~ms}$. 


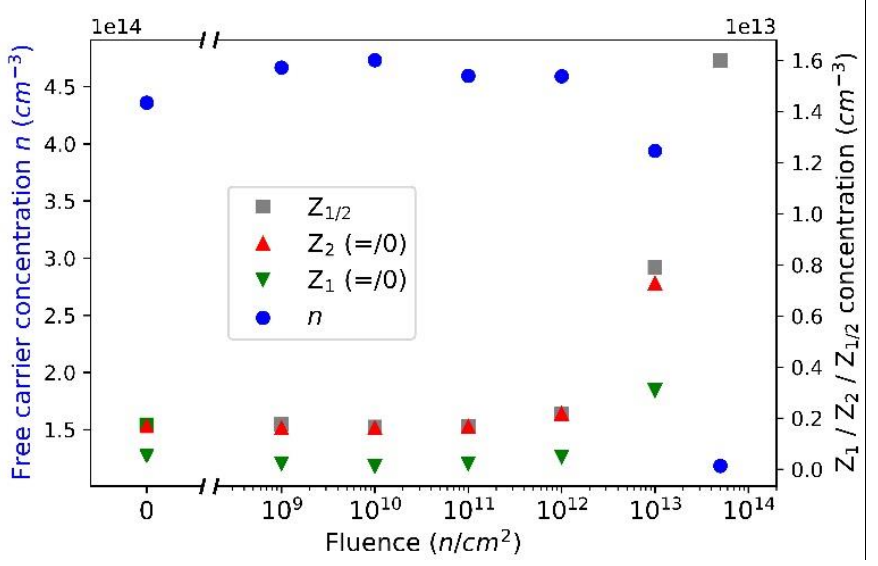

Fig. 3. Influence of neutron irradiation on free carrier, $Z_{1}(=/ 0)$ and $Z_{2}(=/ 0)$ and $Z_{1 / 2}$ concentration. The free carrier concentrations are determined from $C-V$ measurements at $300 \mathrm{~K}$. The $Z_{1}(=/ 0)$ and $Z_{2}(=/ 0)$ concentrations are determined from Laplace DLTS and $C-V$ measurements at $300 \mathrm{~K}$. The $Z_{1 / 2}$ concentrations are determined from DLTS amplitudes (emission rate $50 \mathrm{~s}^{-1}$ ) and $C-V$ measurements at temperature of $Z_{1 / 2}$ peak maximum [32].

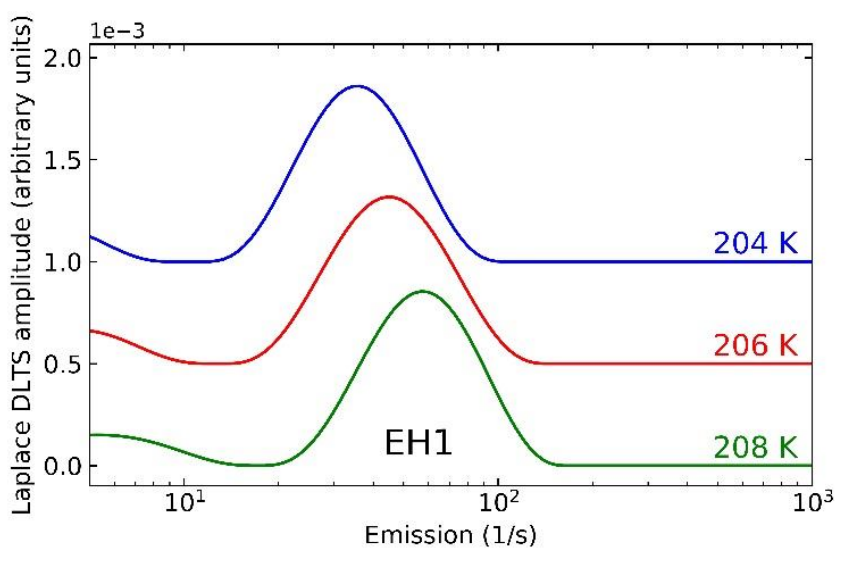

Fig. 4. Laplace DLTS measurements on the sample FN1E13 performed in the temperature range from $194 \mathrm{~K}$ up to $214 \mathrm{~K}$ with $2 \mathrm{~K}$ step (three subsequent measurements are shown). Voltage settings: reverse bias $V_{R}=-10 \mathrm{~V}$, pulse bias $V_{P}=-$ $0.1 \mathrm{~V}$ and pulse width $t_{p}=10 \mathrm{~ms}$. 


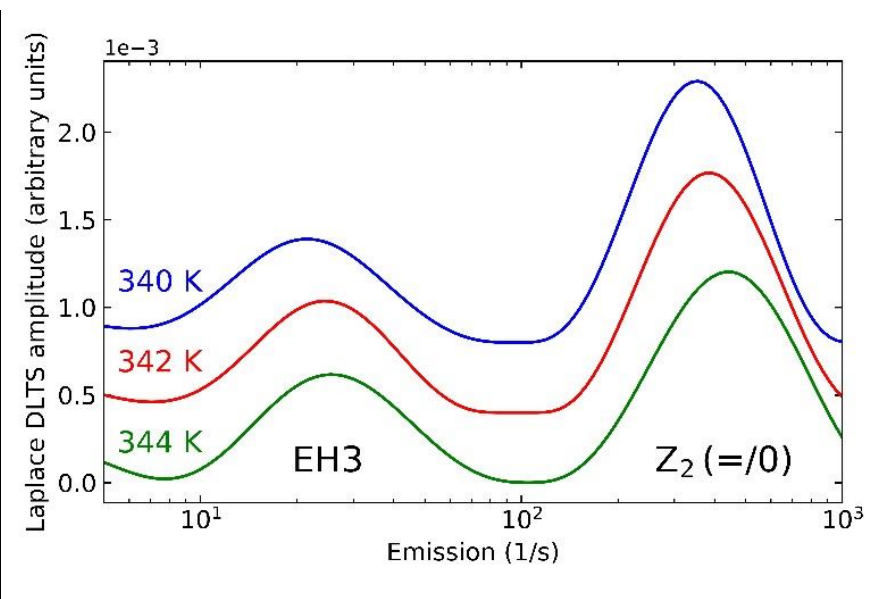

Fig. 5. Laplace DLTS measurements on sample FN1E12 performed in the temperature range from $336 \mathrm{~K}$ up to $370 \mathrm{~K}$ (three subsequent measurements are shown). Voltage settings: reverse bias $V_{R}=-10 \mathrm{~V}$, pulse bias $V_{P}=-0.1 \mathrm{~V}$, pulse width $t_{p}=10 \mathrm{~ms}$. 
Table 1. List of samples with used neutron irradiation settings.

\begin{tabular}{lllll}
\hline $\begin{array}{l}\text { Sample } \\
\text { label }\end{array}$ & $\begin{array}{l}\text { Fluence } \\
\text { (uncertainty) } \\
\left(\mathrm{n} \mathrm{cm}^{-2}\right)\end{array}$ & $\begin{array}{l}\text { Irradiation } \\
\text { time } \\
(\mathrm{s})\end{array}$ & Reactor power & $\begin{array}{l}\text { Sub-Cd flux } \\
\text { (uncertainty) } \\
\left(\mathrm{n} \mathrm{cm}^{-2} \mathrm{~s}^{-1}\right)\end{array}$ \\
\hline FN0 & 0 & 0 & $/$ & $/$ \\
FN1E9 & $1.1 \times 10^{9}(2.9 \%)$ & 30 & $2.5 \mathrm{~W}$ & $3.66 \times 10^{7}(2.7 \%)$ \\
FN1E10 & $1.1 \times 10^{10}(2.7 \%)$ & 300 & $2.5 \mathrm{~W}$ & $3.66 \times 10^{7}(2.7 \%)$ \\
FN1E11 & $9.4 \times 10^{10}(2.9 \%)$ & 273 & $25 \mathrm{~W}$ & $3.44 \times 10^{8}(2.9 \%)$ \\
FN1E12 & $9.4 \times 10^{11}(2.9 \%)$ & 2730 & $25 \mathrm{~W}$ & $3.44 \times 10^{8}(2.7 \%)$ \\
FN1E13 & $1.0 \times 10^{13}(11 \%)$ & 2.73 & $250 \mathrm{~kW}$ & $3.66 \times 10^{12}(2.7 \%)$ \\
FN5E13 & $5.0 \times 10^{13}(2.7 \%)$ & 1365 & $2.5 \mathrm{~kW}$ & $3.66 \times 10^{10}(2.7 \%)$ \\
FN1E14 & $1.0 \times 10^{14}(2.9 \%)$ & 27.3 & $250 \mathrm{~kW}$ & $3.66 \times 10^{12}(2.7 \%)$ \\
\hline
\end{tabular}

Table 2. Activation energies $E_{a}$ and capture cross sections $\sigma$ determined from Laplace DLTS measurements on the FN1E12 and FN1E13 samples (Fig. 2, 4 and 5). Introduction rates $\eta$ [35] of the observed deep levels after the neutron irradiation are included.

\begin{tabular}{|c|c|c|c|c|}
\hline $\begin{array}{l}\text { Deep } \\
\text { level }\end{array}$ & & $\begin{array}{c}E_{a} \\
(e V)\end{array}$ & $\begin{array}{c}\sigma \\
\left(\mathrm{cm}^{2}\right)\end{array}$ & $\begin{array}{c}\eta \\
\left(\mathrm{cm}^{-1}\right)\end{array}$ \\
\hline EH1 & 0.397 & \pm 0.002 & $2 \times 10^{-15}$ & 0.09 \\
\hline$Z_{1}(=/ 0)$ & 0.54 & \pm 0.01 & $7 \times 10^{-16}$ & 0.29 \\
\hline $\mathrm{Z}_{2}(=/ 0)$ & 0.679 & \pm 0.003 & $1 \times 10^{-14}$ & 0.57 \\
\hline $\mathrm{EH} 3$ & 0.70 & \pm 0.01 & $1 \times 10^{-15}$ & 0.80 \\
\hline
\end{tabular}

